\title{
Fixed-target hadron production experiments
}

\author{
Boris A. Popov ${ }^{1,2, a}$ \\ 1 Laboratoire de Physique Nucléaire et de Hautes Energies, 4 place Jussieu, 75005 Paris, France \\ 2 Joint Institute for Nuclear Research, Joliot-Curie 6, 141980 Dubna, Russia
}

\begin{abstract}
Results from fixed-target hadroproduction experiments (HARP, MIPP, NA49 and NA61/SHINE) as well as their implications for cosmic ray and neutrino physics are reviewed. HARP measurements have been used for predictions of neutrino beams in K2K and MiniBooNE/SciBooNE experiments and are also being used to improve predictions of the muon yields in EAS and of the atmospheric neutrino fluxes as well as to help in the optimization of neutrino factory and super-beam designs. Recent measurements released by the NA61/SHINE experiment are of significant importance for a precise prediction of the J-PARC neutrino beam used for the T2K experiment and for interpretation of EAS data. These hadroproduction experiments provide also a large amount of input for validation and tuning of hadron production models in Monte-Carlo generators.
\end{abstract}

\section{Introduction}

The interpretation of extensive air shower (EAS) data - as for instance recorded by the Pierre Auger Observatory [1], KASCADE [2] or IceTop [3] - relies to a large extent on the correct modeling of hadron-air interactions that occur during the shower development. The relevant particle energies span a wide range from primary energies of $\gtrsim 10^{20} \mathrm{eV}$ down to energies of $10^{9} \mathrm{eV}$. Mesons that decay to muons at the ground level and that are detected by these experiments typically originate from low-energy interactions at the late stages of an air shower. Depending on the primary energy and detection distance, the corresponding interaction energies are between 10 and $1000 \mathrm{GeV}$ (see e.g. [4]).

Fixed-target hadron production experiments relevant for cosmic ray and neutrino physics and covering this energy range are briefly reviewed here.

\section{The HARP experiment}

The HARP experiment [5] at the CERN PS was designed to make measurements of hadron yields from a large range of nuclear targets from hydrogen to lead and for incident particle momenta from 1.5 to $15 \mathrm{GeV} / \mathrm{c}$. The main motivations are the measurement of pion yields for a quantitative design of the proton driver of a future neutrino factory and a super-beam [6], a substantial improvement in the calculation of the muon yields in EAS initiated by UHE cosmic rays and of the atmospheric neutrino fluxes [7] and the measurement of particle yields as input for the flux calculation in accelerator neutrino experiments [8], such as K2K [9, 10], MiniBooNE [11] and SciBooNE [12]. In addition to these specific aims, the data provided by HARP are valuable for validation and tuning of hadron production models used in simulation programs. The HARP experiment is described in more detail below

a e-mail: Boris.Popov@cern.ch in order to illustrate some common features of modern hadron production experiments.

To provide a large angular and momentum coverage of the produced charged particles, the HARP experiment makes use of a large-acceptance spectrometer consisting of forward and large-angle detection systems. A detailed description of the experimental apparatus can be found in [5]. The forward spectrometer - based on large area drift chambers [13] and a dipole magnet complemented by a set of detectors for particle identification (PID): a timeof-flight wall [14] (TOFW), a large Cherenkov detector (CHE) and an electromagnetic calorimeter - covers polar angles up to $250 \mathrm{mrad}$ which is well matched to the angular range of interest for the measurement of hadron production to calculate the properties of conventional neutrino beams. The large-angle spectrometer - based on a Time Projection Chamber (TPC) located inside a solenoidal magnet - has a large acceptance in the momentum and angular range for the pions relevant to the production of the muons in a neutrino factory. It covers a large majority of the pions accepted in the focusing system of a typical design. The neutrino beam of a neutrino factory originates from the decay of muons which are, in turn, the decay products of pions.

A full set of data collected by the HARP experiment with solid thin ( $5 \%$ of nuclear interaction length, $\left.\lambda_{I}\right)$ and cryogenic targets have been analyzed and published [1527]. Those results cover all the physics subjects discussed above.

\subsection{Results obtained with the HARP forward spectrometer}

A detailed description of established experimental techniques for the data analysis in the HARP forward spectrometer can be found in Refs. [15-18]. Only a brief summary is given here.

This is an Open Access article distributed under the terms of the Creative Commons Attribution License 4.0, which permits unrestricted use, distribution, and reproduction in any medium, provided the original work is properly cited. 
The absolutely normalized double-differential cross section for a process like $p+$ Target $\rightarrow \pi^{+}+X$ can be expressed in bins of pion kinematic variables in the laboratory frame (momentum, $p_{\pi}$, and polar angle, $\theta_{\pi}$ ), as

$$
\frac{d^{2} \sigma^{\pi^{+}}}{d p d \Omega}\left(p_{\pi}, \theta_{\pi}\right)=\frac{A}{N_{\mathrm{A}} \rho t} \cdot \frac{1}{\Delta p \Delta \Omega} \cdot \frac{1}{N_{\mathrm{pot}}} \cdot N^{\pi^{+}}\left(p_{\pi}, \theta_{\pi}\right),
$$

where $\frac{d^{2} \sigma^{+}}{d p d \Omega}$ is the cross section in $\mathrm{cm}^{2} /(\mathrm{GeV} / \mathrm{c}) / \mathrm{srad}$ for each $\left(p_{\pi}, \theta_{\pi}\right)$ bin covered in the analysis; $\frac{A}{N_{\mathrm{A}} \rho}$ is the reciprocal of the number density of target nuclei; $t$ is the thickness of the target along the beam direction; $\Delta p$ and $\Delta \Omega$ are the bin sizes in momentum and solid angle $(\Delta p=$ $\left.p_{\text {max }}-p_{\text {min }} ; \Delta \Omega=2 \pi\left(\cos \left(\theta_{\text {min }}\right)-\cos \left(\theta_{\max }\right)\right)\right) ; N_{\text {pot }}$ is the number of protons on target after event selection cuts; $N^{\pi^{+}}\left(p_{\pi}, \theta_{\pi}\right)$ is the yield of positive pions in bins of true momentum and angle in the laboratory frame. Equation (1) can be generalized to give the inclusive cross section for a particle of type $\alpha$

$$
\begin{aligned}
& \frac{d^{2} \sigma^{\alpha}}{d p d \Omega}(p, \theta)=\frac{A}{N_{\mathrm{A}} \rho t} \cdot \frac{1}{\Delta p \Delta \Omega} \cdot \frac{1}{N_{\mathrm{pot}}} \cdot M_{p \theta \alpha p^{\prime} \theta^{\prime} \alpha^{\prime}}^{-1} \\
& \cdot N^{\alpha^{\prime}}\left(p^{\prime}, \theta^{\prime}\right),
\end{aligned}
$$

where reconstructed quantities are marked with a prime and $M_{p \theta \alpha p^{\prime} \theta^{\prime} \alpha^{\prime}}^{-1}$ is the inverse of a matrix which fully describes the migrations between bins of true and reconstructed quantities, namely: laboratory frame momentum, $\mathrm{p}$, laboratory frame angle, $\theta$, and particle type, $\alpha$.

There is a background associated with beam protons interacting in materials other than the nuclear target (parts of the detector, air, etc.). These events are subtracted by using data collected without the nuclear target in place after normalization to the same number of protons on target. This procedure is referred to as the "empty target subtraction".

The event selection is performed in the following way: a good event is required to have a single, well reconstructed and identified beam particle impinging on the nuclear target. A downstream trigger in the forward trigger plane (FTP) is also required to record the event, necessitating an additional set of unbiased, pre-scaled triggers for absolute normalization of the cross section. These pre-scaled triggers (e.g. 1/64) are subject to exactly the same selection criteria for a "good" beam particle as the event triggers allowing the efficiencies of the selection to cancel, thus adding no additional systematic uncertainty to the absolute normalization of the result. Secondary track selection criteria have been optimized to ensure the quality of the momentum reconstruction as well as a clean time-offlight measurement while maintaining high reconstruction and particle identification efficiencies $[16,17]$.

The first HARP physics publication [15] reported measurements of the $\pi^{+}$production cross section from a thin $5 \% \quad \lambda_{\mathrm{I}}$ aluminum target at $12.9 \mathrm{GeV} / \mathrm{c}$ proton momentum. This corresponds to the energy of the KEK PS and the target material used by the $\mathrm{K} 2 \mathrm{~K}$ experiment. The results obtained in Ref. [15] were subsequently applied to the final $\mathrm{K} 2 \mathrm{~K}$ neutrino oscillation analysis [10], allowing a significant reduction of the dominant systematic error associated with the calculation of the so-called far-to-near ratio from $5.1 \%$ to $2.9 \%$ (see [15] and [10] for a detailed discussion) and thus an increased $\mathrm{K} 2 \mathrm{~K}$ sensitivity to the oscillation signal.

The next HARP goal was to contribute to the understanding of the MiniBooNE and SciBooNE neutrino fluxes. They are both produced by the Booster Neutrino Beam at Fermilab which originates from protons accelerated to $8.9 \mathrm{GeV} / \mathrm{c}$ by the booster before being collided against a beryllium target. A fundamental input for the calculation of the resulting $v_{\mu}$ flux is the measurement of the $\pi^{+}$cross sections from a thin $5 \% \lambda_{\mathrm{I}}$ beryllium target at exactly $8.9 \mathrm{GeV} / \mathrm{c}$ proton momentum.

These double-differential cross sections were measured in the kinematic range from $0.75 \mathrm{GeV} / \mathrm{c} \leq p_{\pi} \leq$ $6.5 \mathrm{GeV} / \mathrm{c}$ and $0.030 \mathrm{rad} \leq \theta_{\pi} \leq 0.210 \mathrm{rad}$, subdivided into 13 momentum and 6 angular bins [17]. A typical total uncertainty of $9.8 \%$ on the double-differential cross section values and a $4.9 \%$ uncertainty on the total integrated cross section are obtained. These HARP results have been used for neutrino flux predictions [28] in the MiniBooNE [29] and SciBooNE experiments [12].

Sanford and Wang [30] have developed an empirical parametrization for describing the production cross sections of mesons in proton-nucleus interactions (e.g. $\left.\mathrm{p}+\mathrm{A} \rightarrow \pi^{+}+X\right)$. This Sanford-Wang (SW) parametrization has the following functional form:

$$
\begin{aligned}
& \frac{d^{2} \sigma}{d p d \Omega}(p, \theta)=\exp \left[c_{1}-c_{3} \frac{p^{c_{4}}}{p_{\mathrm{b}}^{c_{5}}}\right. \\
& \left.-c_{6} \theta\left(p-c_{7} p_{\mathrm{b}} \cos ^{c_{8}} \theta\right)\right] p^{c_{2}}\left(1-\frac{p}{p_{\mathrm{b}}}\right),
\end{aligned}
$$

where $X$ denotes any system of other particles in the final state; $p_{\mathrm{b}}$ is the proton beam momentum in $\mathrm{GeV} / \mathrm{c}$; $p$ and $\theta$ are the $\pi^{+}$momentum and angle in units of $\mathrm{GeV} / \mathrm{c}$ and radians, respectively; $d^{2} \sigma /(d p d \Omega)$ is expressed in units of $\mathrm{mb} /(\mathrm{GeV} / \mathrm{c} \mathrm{sr}) ; d \Omega \equiv 2 \pi d(\cos \theta)$; and the parameters $c_{1}, \ldots, c_{8}$ are obtained from fits to meson production data. HARP data were fitted using this empirical SW parametrization. This is a useful tool to compare and combine different data sets. A global SW parametrization for forward production of charged pions as an approximation of all the studied datasets is provided in Ref. [26]. It can serve as a tool for quick yields estimates.

The next HARP analysis was devoted to the measurement of the double-differential production cross section of $\pi^{ \pm}$in the collision of $12 \mathrm{GeV} / \mathrm{c}$ protons (see Fig. 1) and pions with a thin $5 \% \lambda_{\mathrm{I}}$ carbon target [18]. These measurements are important for a precise calculation of the atmospheric neutrino flux [7] and for a prediction of the development of EAS [4]. Simulations predict that collisions of protons with a carbon target are very similar to proton interactions with air. This hypothesis could be directly tested with the HARP data. Measurements with cryogenic $\mathrm{O}_{2}$ and $\mathrm{N}_{2}$ targets [19], also shown in Fig. 1, confirm that $\mathrm{p}+\mathrm{C}$ data can indeed be used to predict pion production in $\mathrm{p}+\mathrm{O}_{2}$ and $\mathrm{p}+\mathrm{N}_{2}$ interactions.

It is important to emphasize that a systematic campaign of measurements was performed by HARP: all thin target 

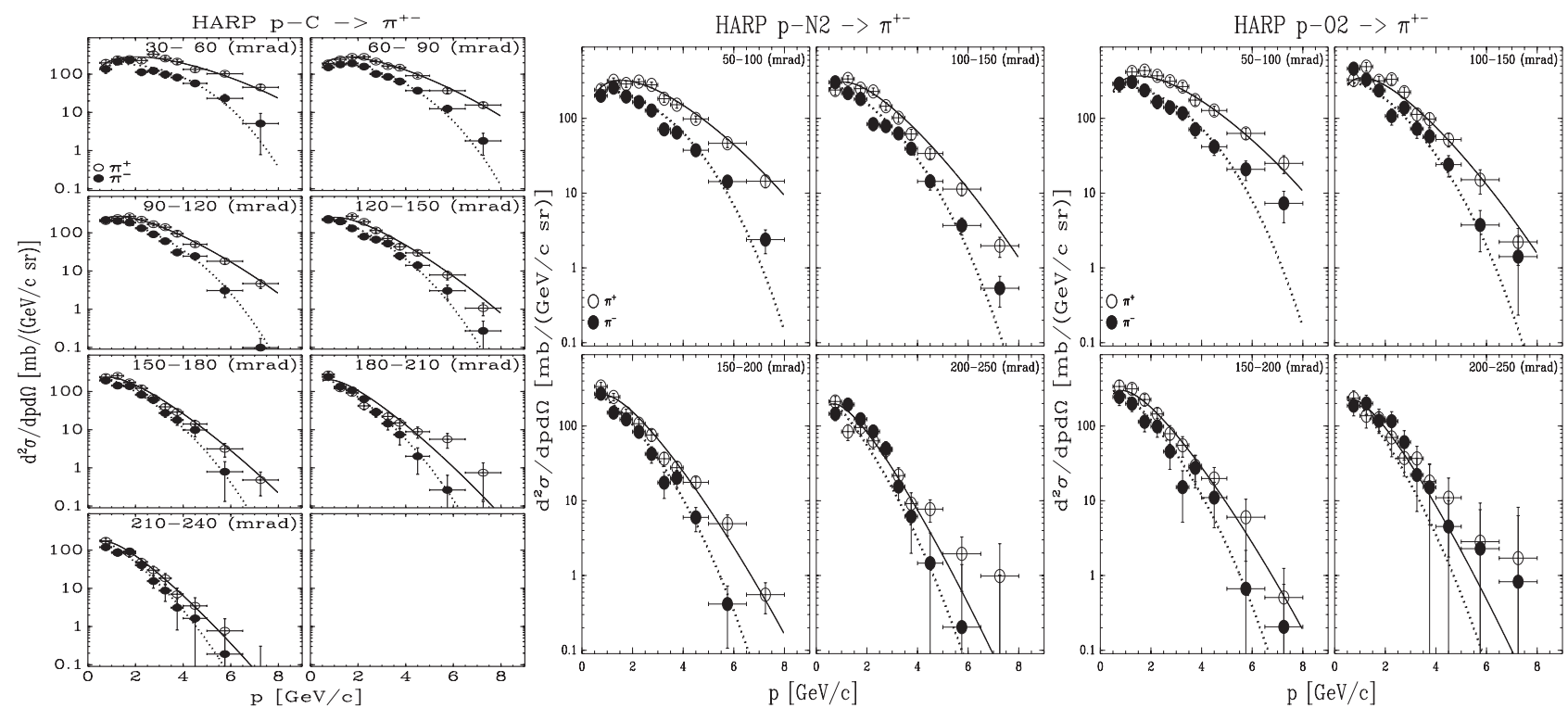

Figure 1. HARP results: measurements of the double-differential production cross sections of $\pi^{+}$(open circles) and $\pi^{-}$(closed circles) from $12 \mathrm{GeV} / \mathrm{c}$ protons on carbon (left), nitrogen (middle) and oxygen (right) targets as a function of pion momentum, $\mathrm{p}$, in bins of pion polar angle, $\theta$, in the laboratory frame. Different panels show different angular intervals. The error bars shown include statistical errors and all (diagonal) systematic errors. The curves show the SW parametrization of Eq. (3) with parameters given in Ref. [18] (solid line for $\pi^{+}$and dashed line for $\pi^{-}$).
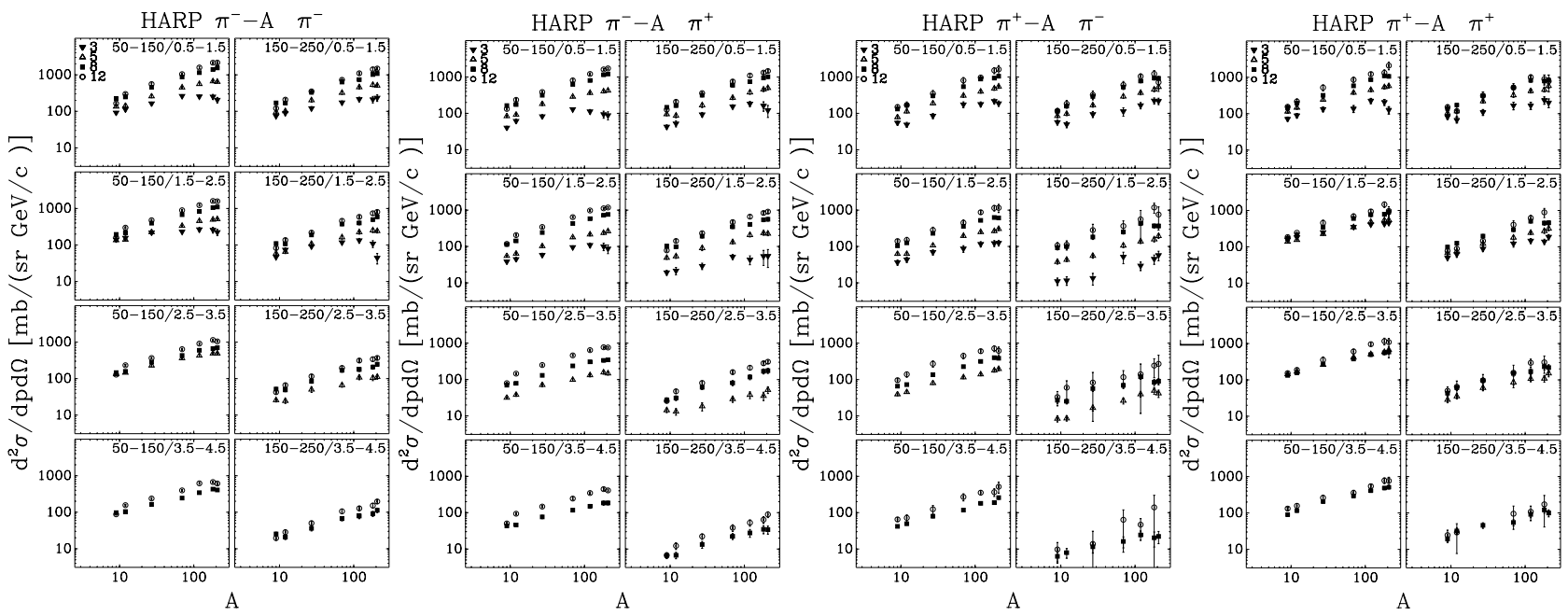

Figure 2. HARP results: the dependence on the atomic number $A$ of the charged pion production yields in $\pi^{-}+\mathrm{Be}, \pi^{-}+\mathrm{C}, \pi^{-}+\mathrm{Al}$, $\pi^{-}+\mathrm{Cu}, \pi^{-}+\mathrm{Sn}, \pi^{-}+\mathrm{Ta}, \pi^{-}+\mathrm{Pb}$ (two left plots) and in $\pi^{+}+\mathrm{Be}, \pi^{+}+\mathrm{C}, \pi^{+}+\mathrm{Al}, \pi^{+}+\mathrm{Cu}, \pi^{+}+\mathrm{Sn}, \pi^{+}+\mathrm{Ta}, \pi^{+}+\mathrm{Pb}$ (two right plots) interactions averaged over two forward angular regions $(0.05 \mathrm{rad} \leq \theta<0.15 \mathrm{rad}$ and $0.15 \mathrm{rad} \leq \theta<0.25 \mathrm{rad})$ and four momentum regions $(0.5 \mathrm{GeV} / c \leq p<1.5 \mathrm{GeV} / c, 1.5 \mathrm{GeV} / c \leq p<2.5 \mathrm{GeV} / c, 2.5 \mathrm{GeV} / c \leq p<3.5 \mathrm{GeV} / c$ and $3.5 \mathrm{GeV} / c \leq p<4.5 \mathrm{GeV} / c)$, for the four different incoming beam momenta $(3,5,8$ and $12 \mathrm{GeV} / \mathrm{c})[20]$.

data taken with pion beams are analyzed and published now [20]. Figure 2 shows the dependence on the atomic number $A$ of the forward $\pi^{ \pm}$yields in $\pi^{ \pm}+A$ $(A=\mathrm{Be}, \mathrm{C}, \mathrm{Al}, \mathrm{Cu}, \mathrm{Sn}, \mathrm{Ta}, \mathrm{Pb})$ interactions averaged over two forward angular regions $(0.05 \mathrm{rad} \leq \theta<$ $0.15 \mathrm{rad}$ and $0.15 \mathrm{rad} \leq \theta<0.25 \mathrm{rad})$ and four momentum regions $(0.5 \mathrm{GeV} / c \leq p<1.5 \mathrm{GeV} / c, 1.5 \mathrm{GeV} / c \leq$ $p<2.5 \mathrm{GeV} / c, \quad 2.5 \mathrm{GeV} / c \leq p<3.5 \mathrm{GeV} / c \quad$ and $3.5 \mathrm{GeV} / c \leq p<4.5 \mathrm{GeV} / c)$, for the four different incoming beam momenta $(3,5,8$ and $12 \mathrm{GeV} / \mathrm{c})$. Similar results were also obtained for incoming protons [26]. Moreover, results on forward proton production in $\mathrm{p}+A$ and $\pi^{ \pm}+A$ interactions were also published [27].

\subsection{Results obtained with the HARP large-angle spectrometer}

First results on the measurements of the double-differential cross section for the production of charged pions in $\mathrm{p}$ $A$ collisions emitted at large angles from the incoming beam direction were obtained for the full set of available nuclear targets using an initial part of the accelerator spill to avoid distortions in the TPC [21-23]. The pions were produced by proton beams in a momentum range from $3 \mathrm{GeV} / \mathrm{c}$ to $12 \mathrm{GeV} / \mathrm{c}$ hitting a target with a thickness of $5 \% \lambda_{\mathrm{I}}$. The angular and momentum range covered by the experiment $(100 \mathrm{MeV} / \mathrm{c} \leq p<800 \mathrm{MeV} / \mathrm{c}$ and 
$0.35 \mathrm{rad} \leq \theta<2.15 \mathrm{rad})$ is of particular importance for the design of a neutrino factory. Track recognition, momentum determination and particle identification were all performed based on the measurements made with the TPC (see [21] for more details).

Full spill data were analyzed and published for incoming protons [24] and pions [25], after the development of a special algorithm to correct for the TPC dynamic distortions [31] and validation of the TPC performance with benchmarks based on real data [32],

An additional comparison was also performed on the pion yields measured with the short $\left(5 \% \lambda_{\mathrm{I}}\right)$ and long $\left(100 \% \lambda_{\mathrm{I}}\right)$ nuclear targets [33].

The advantage of all these measurements is that they were performed with the same detector, thus related systematic uncertainties are minimized.

All HARP measurements described above were compared with predictions of Monte-Carlo models available within GEANT4 [34] and MARS [35], as well as with the FLUKA [36] and GIBUU [37] models. Some models provide a reasonable description of HARP data in some regions (see e.g. [18-27] and technical notes [38]), while there is no model which would describe all aspects of the data.

Tables with HARP results in text format are available from the DURHAM database [39].

\section{The MIPP experiment}

The MIPP (Main Injector Particle Production) experiment has been perfomed at Fermilab. The primary goals were to measure particle production using $\pi^{ \pm}, \mathrm{K}^{ \pm}$and proton beams between $5 \mathrm{GeV} / \mathrm{c}$ and $120 \mathrm{GeV} / \mathrm{c}$ with various targets, such as liquid hydrogen and nitrogen, $\mathrm{Be}, \mathrm{C}, \mathrm{Cu}$ and $\mathrm{Pb}$, to study non-perturbative particle dynamics. In particular, the aim was to measure particle production off the NuMI target using $120 \mathrm{GeV} / \mathrm{c}$ protons in order to improve the precision of the neutrino flux predictions in the running NuMI experiments. The corresponding results on the production of charged pions off the NuMI target have recently been published [40].

Particle identification capabilities of the MIPP spectrometer include a combination of TPC $(d E / d x)$, timeof-flight, multi-cell Cherenkov and RICH detectors, see e.g. $[41,42]$.

\section{The NA49 experiment}

The NA49 experiment at CERN SPS [43] has performed a detailed analysis of hadronic interactions in p+p@158GeV/c [44] and p+C@158GeV/c [45] by measuring the production properties of charged pions and kaons, (anti-)protons and neutrons. Typical total (stat.+syst.) error of these measurements are below $10 \%$ [46]. An example of the measured invariant cross section

$$
f\left(x_{F}, p_{T}\right)=E\left(x_{F}, p_{T}\right) \cdot \frac{d^{3} \sigma}{d p^{3}}\left(x_{F}, p_{T}\right)
$$

as a function of $p_{T}$ at fixed $x_{F}$ for charged pions produced in $\mathrm{p}+\mathrm{C}$ collisions at $158 \mathrm{GeV} / \mathrm{c}$ is shown in Fig. 3. The
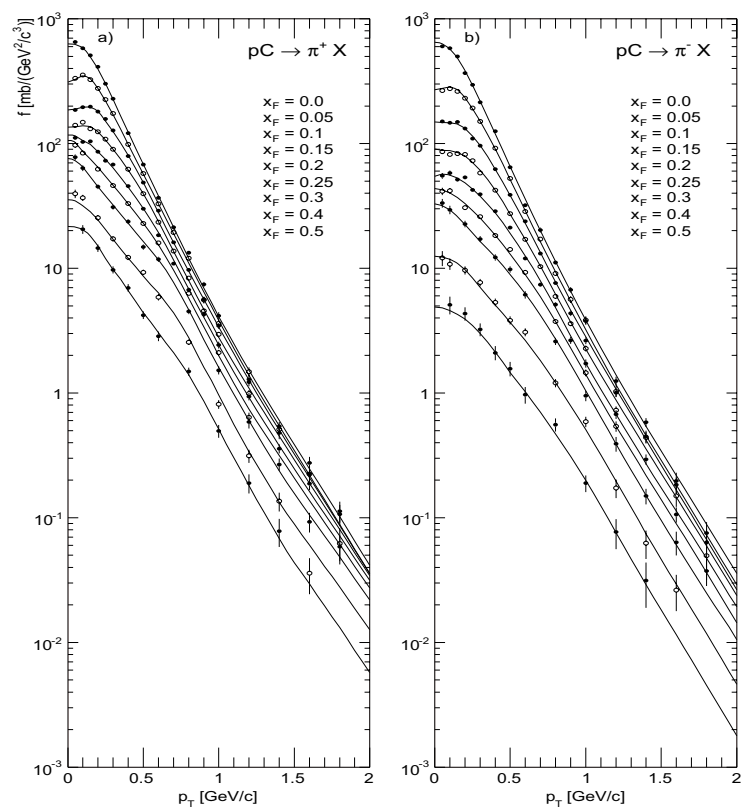

Figure 3. NA49 results: Invariant cross section as a function of $p_{T}$ at fixed $x_{F}$ for a) $\pi^{+}$and b) $\pi^{-}$produced in $\mathrm{p}+\mathrm{C}$ collisions at $158 \mathrm{GeV} / \mathrm{c}$ [45].

NA49 hadron production measurements were used for the prediction of (anti-)neutrino fluxes in the MINOS [47] and MINERvA [48] experiments as well as for improved calculations of atmospheric neutrino fluxes [7].

\section{The NA61/SHINE experiment}

The physics program of the NA61 or SHINE (where SHINE $\equiv$ SPS Heavy Ion and Neutrino Experiment) experiment at the CERN SPS [49] consists of three main subjects. In the first stage of data taking measurements of hadron production in hadron-nucleus interactions needed for neutrino (T2K [50]) and cosmic ray (Pierre Auger [1] and KASCADE [2]) experiments were performed. Later stages of the NA61/SHINE experiment are devoted to a study of hadron production in proton-proton and protonnucleus interactions with high statistics needed for a better understanding of high $p_{T}$ production at SPS energies. Finally, the energy dependence of hadron production properties is being measured in $\mathrm{p}+\mathrm{p}$ [52], $\mathrm{p}+\mathrm{Pb}$ interactions as well as in nucleus-nucleus collisions, with the aim to identify the properties of the onset of deconfinement and find evidence for the critical point of strongly interacting matter [53]. A summary of $h+A$ data recorded by NA61/SHINE so far is presented in Table 1.

The NA61/SHINE apparatus [54] is a large acceptance spectrometer at the CERN SPS for the study of the hadronic final states produced in interactions of various beam particles ( $\pi, \mathrm{p}$, ions) with a variety of fixed targets at the SPS energies. The main components of the current detector were constructed and used by the NA49 experiment [43]. The main tracking devices are four large volume TPCs. Two of them, the vertex TPCs (VTPC-1 and VTPC-2), are located in the magnetic field of two super-conducting dipole magnets (maximum bending power of $9 \mathrm{Tm}$ ) and two others (MTPC-L and MTPC-R) 
Table 1. A summary of h+A data recorded by NA61/SHINE and a status of the analysis as of summer 2014.

\begin{tabular}{|c|c|c|c|c|}
\hline beam & target & year & triggers $\times 10^{6}$ & status of the analysis \\
\hline \multirow{5}{*}{$\begin{array}{l}\text { protons } \\
\text { at } 31 \mathrm{GeV} / c\end{array}$} & \multirow{2}{*}{$\begin{array}{c}\text { thin } C \text { target } \\
2 \mathrm{~cm}\left(0.04 \lambda_{I}\right)\end{array}$} & 2007 & 0.7 & published: $\pi^{ \pm}$[55], $\mathrm{K}^{+}$[56], $\mathrm{K}_{S}^{0}, \Lambda$ [64] \\
\hline & & 2009 & 5.4 & preliminary $\pi^{ \pm}, \mathrm{K}^{ \pm}, \mathrm{p}, \mathrm{K}_{S}^{0}, \Lambda$ results $[62]$ \\
\hline & \multirow{3}{*}{$\begin{array}{c}\text { T2K replica } \\
\text { target } \\
90 \mathrm{~cm}\left(1.9 \lambda_{I}\right)\end{array}$} & 2007 & 0.2 & published: $\pi^{ \pm}[74]$ \\
\hline & & 2009 & 2.8 & preliminary $\pi^{ \pm}$results [75] \\
\hline & & 2010 & $\sim 10$ & 1-st round of calibration completed \\
\hline$\pi^{-}$at $158 \mathrm{GeV} / \mathrm{c}$ & thin $\mathrm{C}$ target & 2009 & 5.5 & preliminary $h^{ \pm}$results $[71,72]$ \\
\hline$\pi^{-}$at $350 \mathrm{GeV} / \mathrm{c}$ & thin $\mathrm{C}$ target & 2009 & 4.6 & preliminary $h^{ \pm}$results $[71,72]$ \\
\hline protons at $20 \mathrm{GeV} / \mathrm{c}$ & & 2009 & 2.2 & \\
\hline protons at $31 \mathrm{GeV} / \mathrm{c}$ & liquid & 2009 & 3.1 & \\
\hline protons at $40 \mathrm{GeV} / \mathrm{c}$ & hydrogen & 2009 & 5.2 & published: $\pi^{-}$[52], preliminary: $\pi^{ \pm}, \mathrm{K}^{ \pm}, \mathrm{p}$ [53] \\
\hline protons at $80 \mathrm{GeV} / \mathrm{c}$ & target & 2009 & 4.5 & \\
\hline protons at $158 \mathrm{GeV} / \mathrm{c}$ & (LHT) & 2009 & 3.5 & \\
\hline protons at $13 \mathrm{GeV} / \mathrm{c}$ & LHT & 2011 & 1.4 & under calibration \\
\hline protons at $158 \mathrm{GeV} / \mathrm{c}$ & LHT & 2010 & 44. & under calibration \\
\hline protons at $158 \mathrm{GeV} / \mathrm{c}$ & LHT & 2011 & 15. & under calibration \\
\hline protons at $158 \mathrm{GeV} / \mathrm{c}$ & $\mathrm{Pb}$ & 2012 & 4.5 & under calibration \\
\hline
\end{tabular}
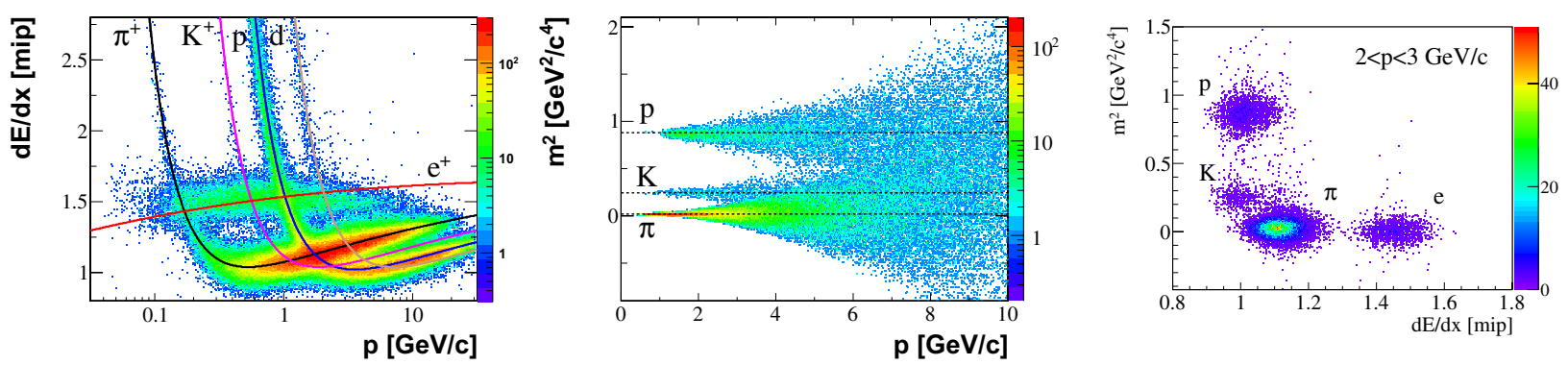

Figure 4. Examples of PID capabilities of the NA61/SHINE spectrometer for positively charged particles. Left: specific energy loss in the TPCs as a function of momentum. Curves show parameterizations of the mean $d E / d x$ calculated for different particle species. Middle: mass squared, derived from the ToF-F measurement and the fitted path length and momentum, versus momentum. The lines show the expected mass squared values for different particles. Right: example of two-dimensional $m^{2}-d E / d x$ plot for the momentum range $2-3 \mathrm{GeV} / c$. Four clear accumulations corresponding to positrons, pions, kaons and protons are observed. The combination of both $m^{2}$ and $d E / d x$ measurements provides close to $100 \%$ purity in the pion selection over the whole momentum range.

are positioned downstream of the magnets symmetrically with respect to the beam line. One additional small TPC, the so-called gap TPC (GTPC), is installed on the beam axis between the vertex TPCs. The setup is supplemented by time of flight detector arrays two of which (ToF-L/R) were inherited from NA49 and can provide a time measurement resolution of $\sigma_{\text {tof }} \leq 90 \mathrm{ps}$. A new forward time of flight detector (ToF-F) with a time resolution of $\sim 120 \mathrm{ps}$ was constructed in order to extend the acceptance of the NA61/SHINE setup for pion and kaon identification as required for the T2K measurements. The particle identification in NA61/SHINE is based on the differential energy loss $\mathrm{dE} / \mathrm{dx}$ measured in the TPCs combined with the mass squared measurements based on the ToF information, see Fig. 4.

Two carbon (isotropic graphite) targets were used for T2K-related measurements: 1 ) a $2 \mathrm{~cm}$-long target (about $4 \% \lambda_{\mathrm{I}}$ ) with density $\rho=1.84 \mathrm{~g} / \mathrm{cm}^{3}$, the so-called thin target; 2) a $90 \mathrm{~cm}$ long cylinder of $2.6 \mathrm{~cm}$ diameter (about $\left.1.9 \lambda_{\mathrm{I}}\right)$, the so-called $\mathrm{T} 2 \mathrm{~K}$ replica target (RT) with density $\rho=1.83 \mathrm{~g} / \mathrm{cm}^{3}$.

\subsection{Thin-target results}

The NA61/SHINE experiment was approved at CERN in June 2007. The first pilot run was performed during
October 2007. In total about $670 \mathrm{k}$ events with the thin target, $230 \mathrm{k}$ events with the $\mathrm{T} 2 \mathrm{~K}$ replica target and $80 \mathrm{k}$ events without target (empty target events) were registered. Using these data interaction cross sections and charged pion spectra in $\mathrm{p}+\mathrm{C}$ interactions at $31 \mathrm{GeV} / \mathrm{c}$ were first measured [55]. Such measurements are required to improve predictions of the neutrino flux for the T2K long baseline neutrino oscillation experiment in Japan [50,51].

For normalization and cross section measurements NA61/SHINE adopted the same procedure as the one developed by the NA49 Collaboration $[57,58]$.

Crucial for this analysis is the identification of the produced charged pions. Depending on the momentum interval, different approaches have been adopted, which lead also to different track selection criteria. The task is facilitated for the negatively charged pions, by the observation that more than $90 \%$ of primary negatively charged particles produced in $\mathrm{p}+\mathrm{C}$ interactions at this energy are $\pi^{-}$, thus the analysis of $\pi^{-}$spectra can also be carried out without additional particle identification.

Three analysis methods were developed to obtain charged pion spectra. These are: 1) analysis of $\pi^{-}$ mesons via measurements of negatively charged particles ( $h^{-}$analysis) [59]; 2) analysis of $\pi^{+}$and $\pi^{-}$mesons identified via $d E / d x$ measurements in the TPCs $(d E / d x$ analysis at low momentum) [60] and 3) analysis of $\pi^{+}$ 

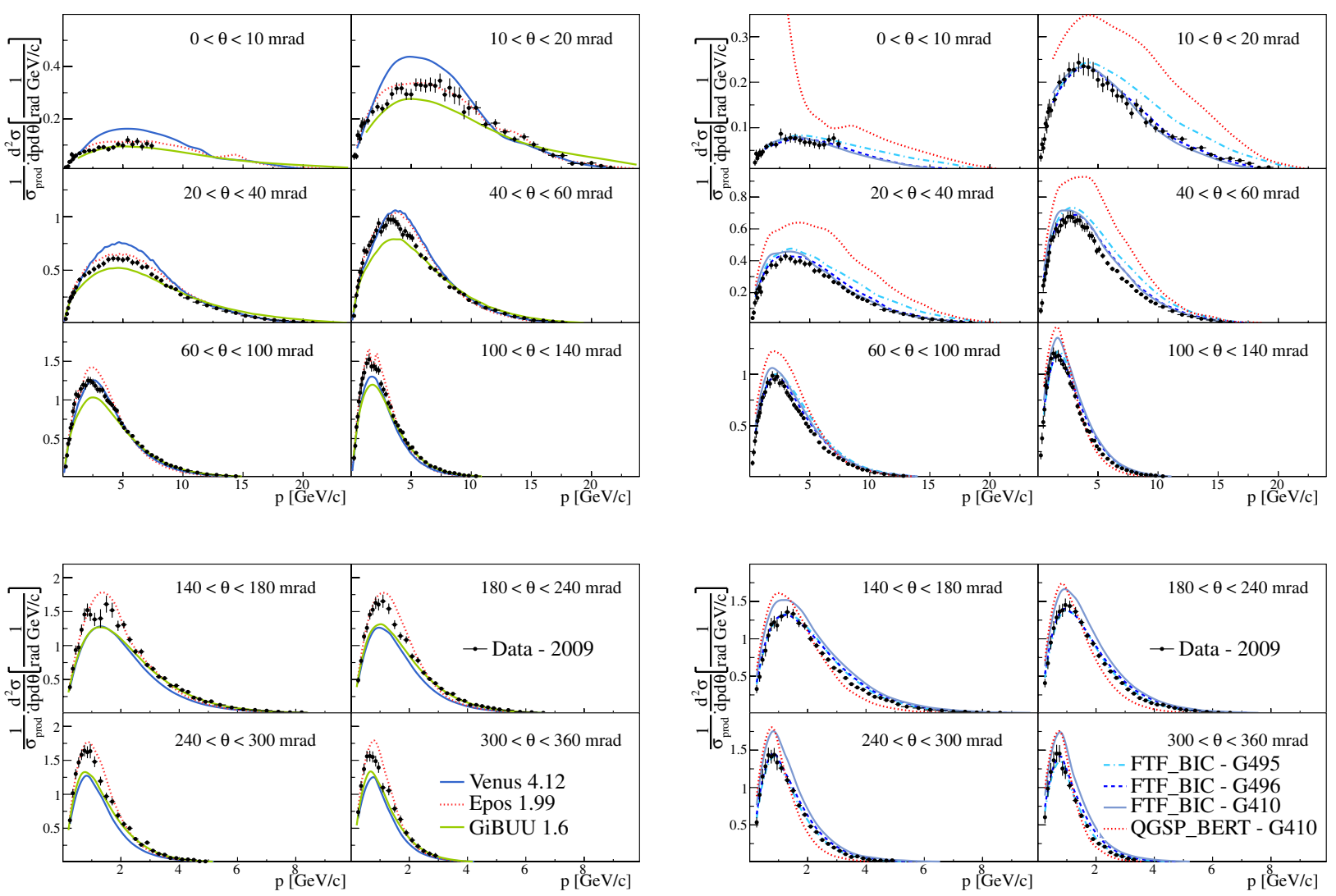

Figure 5. NA61/SHINE preliminary results: laboratory momentum distributions of $\pi^{+}$(left) and $\pi^{-}$(right) mesons in production $\mathrm{p}+\mathrm{C}$ interactions at $31 \mathrm{GeV} / \mathrm{c}$ in different intervals of polar angle $(\theta)$. The spectra are normalized to the mean $\pi^{ \pm}$multiplicity in all production $\mathrm{p}+\mathrm{C}$ interactions. Error bars indicate statistical and systematic uncertainties added in quadrature. Predictions of hadron production models - VENUS 4.12, EPOS 1.99, GIBUU 1.6 and selected GEANT4 physics lists (QGSP_BERT and FTF_BIC in different releases from 4.9.5 till 4.10) - are also indicated.

and $\pi^{-}$mesons identified via time-of-flight and $d E / d x$ measurements in the ToF-F and TPCs, respectively (tof $d E / d x$ analysis) [61]. Each analysis yields fully corrected pion spectra with independently calculated statistical and systematic errors. The spectra were compared in overlapping phase-space domains to check their consistency. Complementary domains were combined to reach maximum acceptance.

Developed analysis techniques have been applied to a large sample $\left(5.4 \times 10^{6}\right.$ triggers $)$ of $\mathrm{p}+\mathrm{C} @ 31 \mathrm{GeV} / \mathrm{c}$ thintarget data collected during the 2009 run. New preliminary results have been released and are being prepared for publication [62], a brief summary is presented here.

The measured inelastic and production cross sections in proton-carbon interactions at $31 \mathrm{GeV} / \mathrm{c}$ are $261.3 \pm$ 2.8 (stat) $\pm 2.4($ det $) \pm 0.3$ (model) $\mathrm{mb}$ and $233.5 \pm 2.8$ (stat) $\pm 2.4($ det $) \pm 3.6$ (model) mb, respectively.

Inclusive production cross sections for negatively and positively charged pions are presented as a function of laboratory momentum in 10 intervals of the laboratory polar angle covering the range from 0 up to $360 \mathrm{mrad}$. The spectra for $\pi^{+}$and $\pi^{-}$are plotted in Fig. 5. For the purpose of a comparison of the data with model predictions the spectra were normalized to the mean $\pi^{ \pm}$multiplicity in all production interactions. This avoids uncertainties due to the different treatment of quasi-elastic interactions in models as well as problems due to the absence of predictions for inclusive cross sections. A clear advantage of NA61/SHINE is that it fully covers the kinematic phasespace of interest for $\mathrm{T} 2 \mathrm{~K}$.

It is interesting to compare the $\pi^{ \pm}$spectra in $\mathrm{p}+\mathrm{C}$ interactions at $31 \mathrm{GeV} / \mathrm{c}$ to the predictions of event generators of hadronic interactions. Some of the models are frequently used for the interpretation of cosmic ray data, i.e. VENUS4.12 [65], EPOS1.99 [66], others are heavily used for accelerator-based experiments, e.g. GEANT4 toolkit [34]. The results are presented in Fig. 5. Many more model comparisons can be found in the technical notes [70].

The tof $-d E / d x$ analysis was applied also to the set of 2007 pilot data in order to perform measurements of differential production cross sections of positively charged kaons in $\mathrm{p}+\mathrm{C}$ interactions at $31 \mathrm{GeV} / \mathrm{c}$ [56]. New results are now available from the analysis of the full statistics collected during the 2009 run. A much higher statistics available in the 2009 data makes it possible to use finer $\{p, \theta\}$ binning. An improved statistics of the 2009 data also allows for the first measurements of negatively charged kaons within NA61/SHINE. The corresponding results are shown in Fig. 6 (left) together with model predictions. The knowledge of kaon production is crucial for a precise 

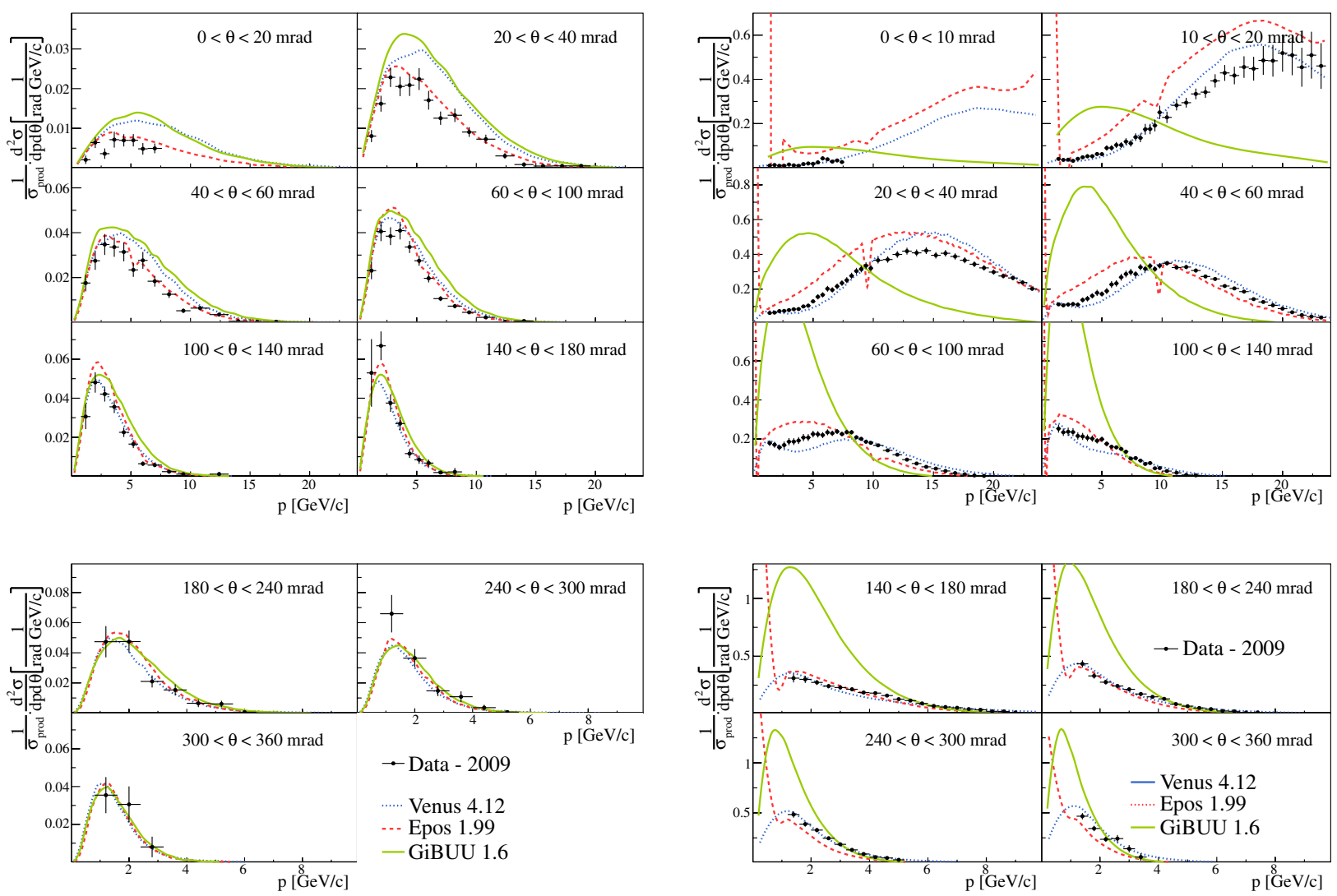

Figure 6. NA61/SHINE preliminary results: comparison of measured $K^{-}$(left) and proton (right) spectra with model predictions. Distributions are normalized to the mean $K^{-}$(p) multiplicity in all production $\mathrm{p}+\mathrm{C}$ interactions. The vertical error bars on the data points show the total (stat. and syst.) uncertainty. The horizontal error bars indicate the bin size in momentum.

prediction of the intrinsic electron neutrino component and the high energy tail of the T2K beam [51].

Preliminary results for proton yields were also obtained, see Fig. 6 (right). Clearly, some hadron production models have difficulties in describing these measurements.

The analysis of neutral strange particles $\left(K_{s}^{0}\right.$ and $\left.\Lambda\right)$ production using $\mathrm{V}^{0}$-like topology of their decays has been developed [59,63] and the results are now published [64]. New preliminary results from the analysis of the 2009 dataset have been also released recently.

During the 2009 run, NA61/SHINE also recorded data with negatively charged pion beams at 158 and $350 \mathrm{GeV} / \mathrm{c}$ on a thin carbon target to provide particle-production data for the tuning of hadronic interaction models used in air-shower simulations and first preliminary results have already been released $[71,72]$. They are summarized below.

The results on the production of charged hadrons in $\pi^{-}+\mathrm{C}$ interactions at 158 and $350 \mathrm{GeV} / \mathrm{c}$ are presented in Fig. 7. These spectra were obtained within a fiducial phase space of the NA61/SHINE detector, for which the detection and selection efficiency for charged tracks is close to unity, and corrected for feed-down and track loss using the average correction predicted by the VENUS and EPOS event generators after simulation of the detector response. The trigger bias is corrected for by studying the track loss in a sub-sample of unbiased beam-trigger data. Only phase-space regions for which the overall model correction stays below $20 \%$ and for which the total systematic uncertainty is smaller than $20 \%$ are displayed in Fig. 7. The uncertainties shown are the total uncertainties including the statistical uncertainty and systematics from the model correction, normalization, trigger bias, calibration and track topology.

This extensive preliminary data set is already useful to judge the quality of event generators used in airshower simulations. Comparisons to the predictions of the EPOS 1.99 model and to the FTF_BIC physics list of GEANT4.9.5 are shown in Figs. 8 and 9, respectively. As can be seen, the EPOS 1.99 generator significantly underestimates the charged-hadron production at large transverse momenta.

It is interesting to note that the NA61/SHINE data have already been used to improve predictions of several hadron production models, e.g. URQMD1.3.1 [67,68] and Fritiof-based models [69].

\subsection{T2K replica-target results}

Measurements of pion emission from the T2K RT were also performed [73].

The main motivation for measurements of hadron emission from a replica of the $\mathrm{T} 2 \mathrm{~K}$ target is to reduce the systematic uncertainties on the prediction of the initial neutrino flux originating from re-interactions in the long target. 


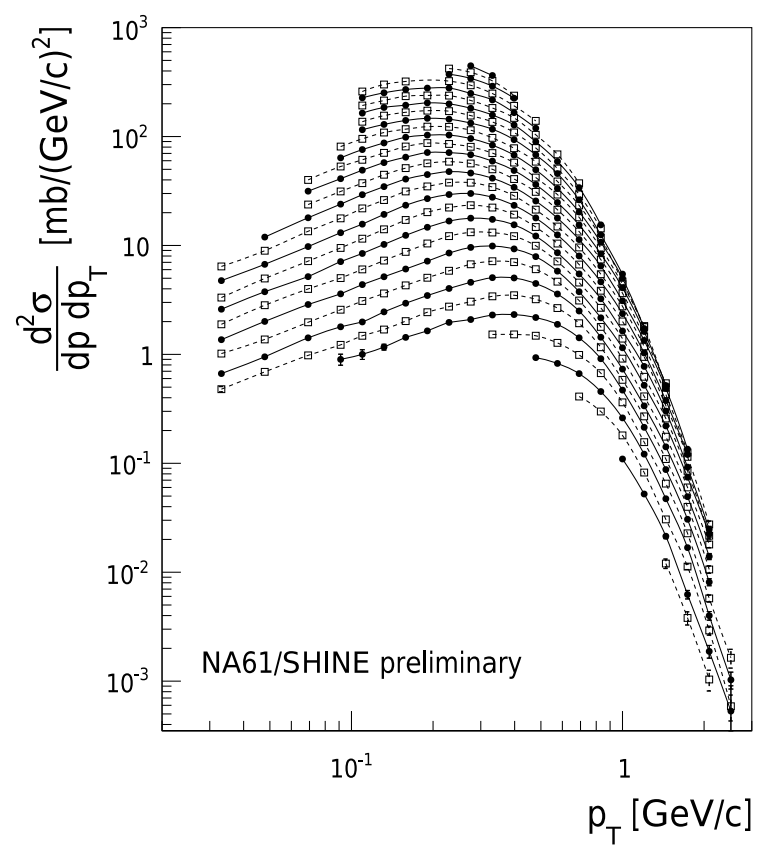

(a) $h^{-}$at $158 \mathrm{GeV} / \mathrm{c}$

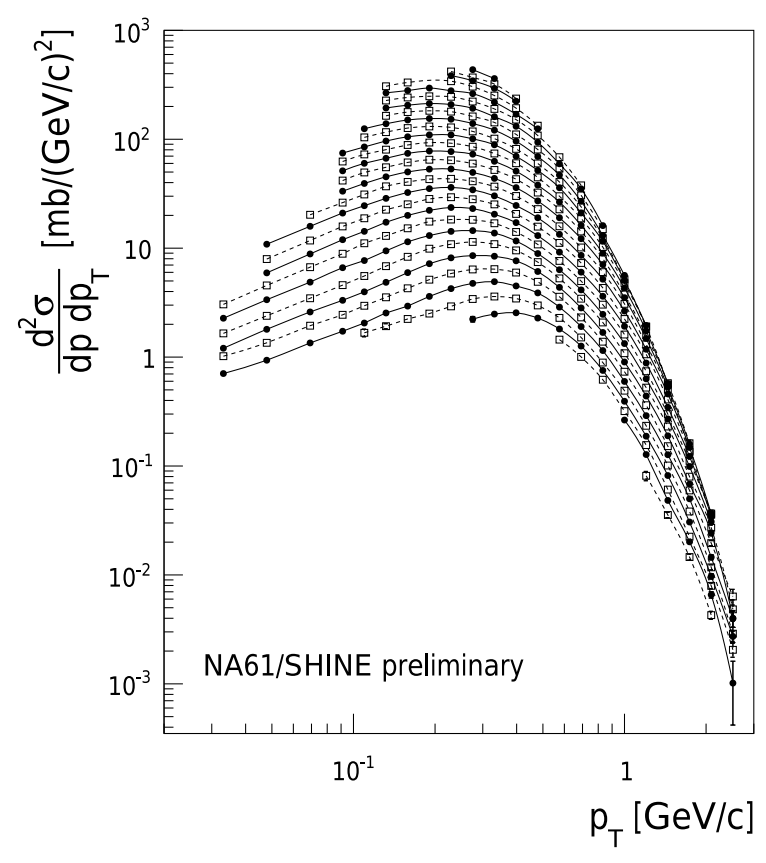

(c) $h^{-}$at $350 \mathrm{GeV} / \mathrm{c}$

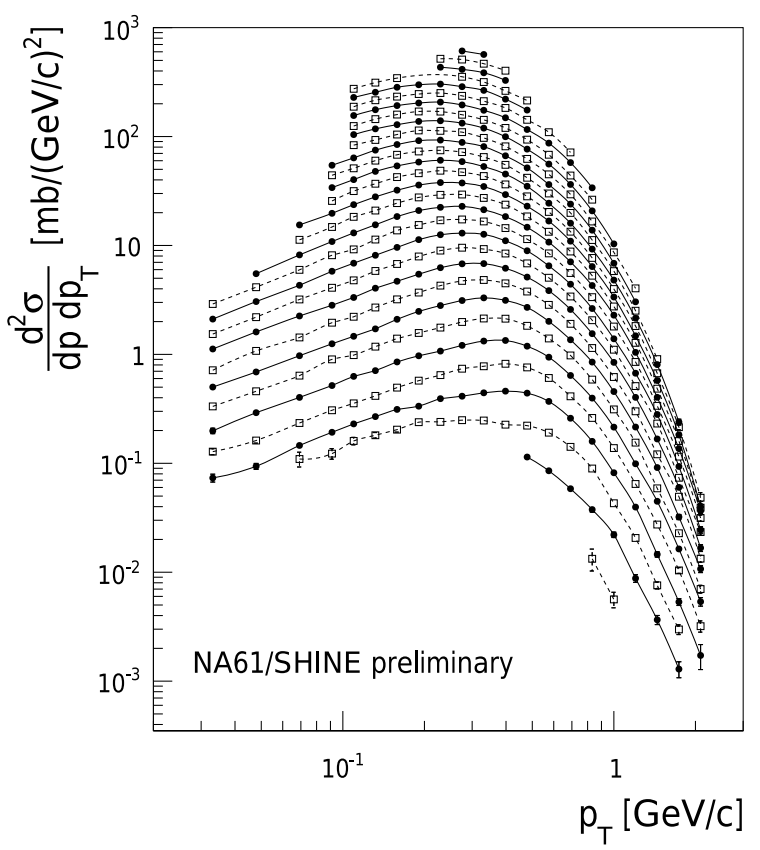

(b) $h^{+}$at $158 \mathrm{GeV} / \mathrm{c}$

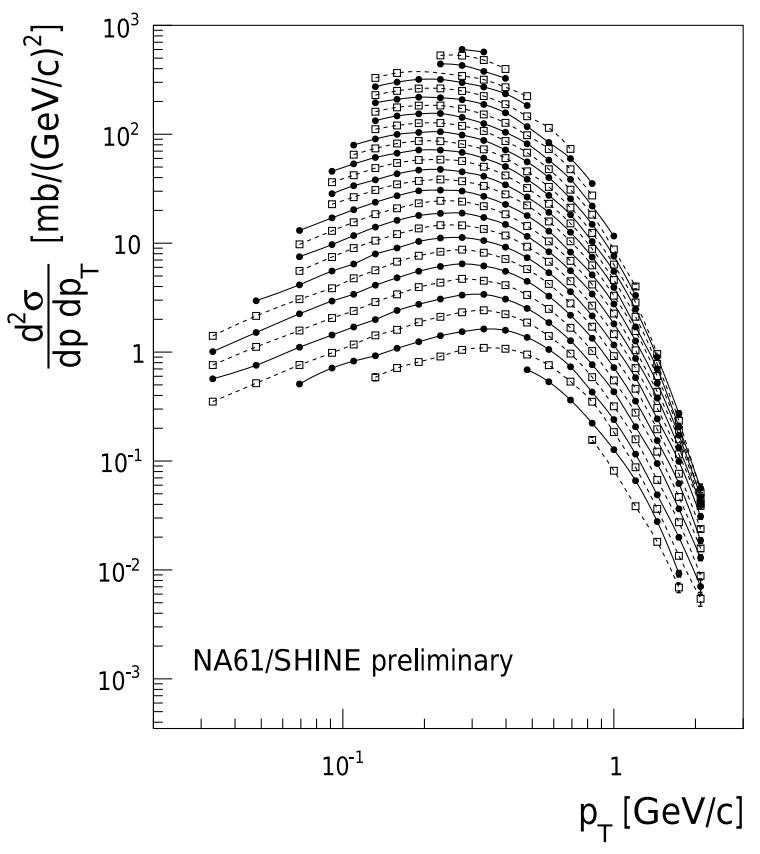

(d) $h^{+}$at $350 \mathrm{GeV} / \mathrm{c}$

Figure 7. NA61/SHINE preliminary results: inclusive $p_{\mathrm{T}}$-spectra of charged hadrons produced in $\pi^{-}+\mathrm{C}$ interactions at 158 and $350 \mathrm{GeV} / \mathrm{c}$. In each figure, $p$ goes from 0.6 to $121 \mathrm{GeV} / \mathrm{c}$ in steps of $\log p /(\mathrm{GeV} / \mathrm{c})=0.08$ from top to bottom.

The first long-target analysis used the low-statistics data collected in 2007 [74]. The goal was to set the ground for the on-going analysis of high-statistics NA61/SHINE data with the replica of the T2K target. It demonstrated that high-quality long-target data were successfully taken with the NA61/SHINE apparatus for T2K, and that such data can effectively be used to constrain the T2K neutrino flux predictions. It is important to stress that this was the first complete example of application of long-target data for neutrino flux predictions.
Figure 10 illustrates the preliminary results obtained in the analysis of a larger-statistics 2009 p+RT@31GeV/c dataset [75]. Fully corrected yields of $\pi^{+}$for two bins of polar angle $([100,140] \mathrm{mrad}$ and $[140,180] \mathrm{mrad})$ measured in 6 longitudinal intervals are shown and compared to FLUKA2011 predictions.

The data presented in Refs. $[55,56,74]$ provide important information used for improved calculation of the T2K neutrino flux [51]. New NA61/SHINE measurements described here will allow to reach even a better precision. 

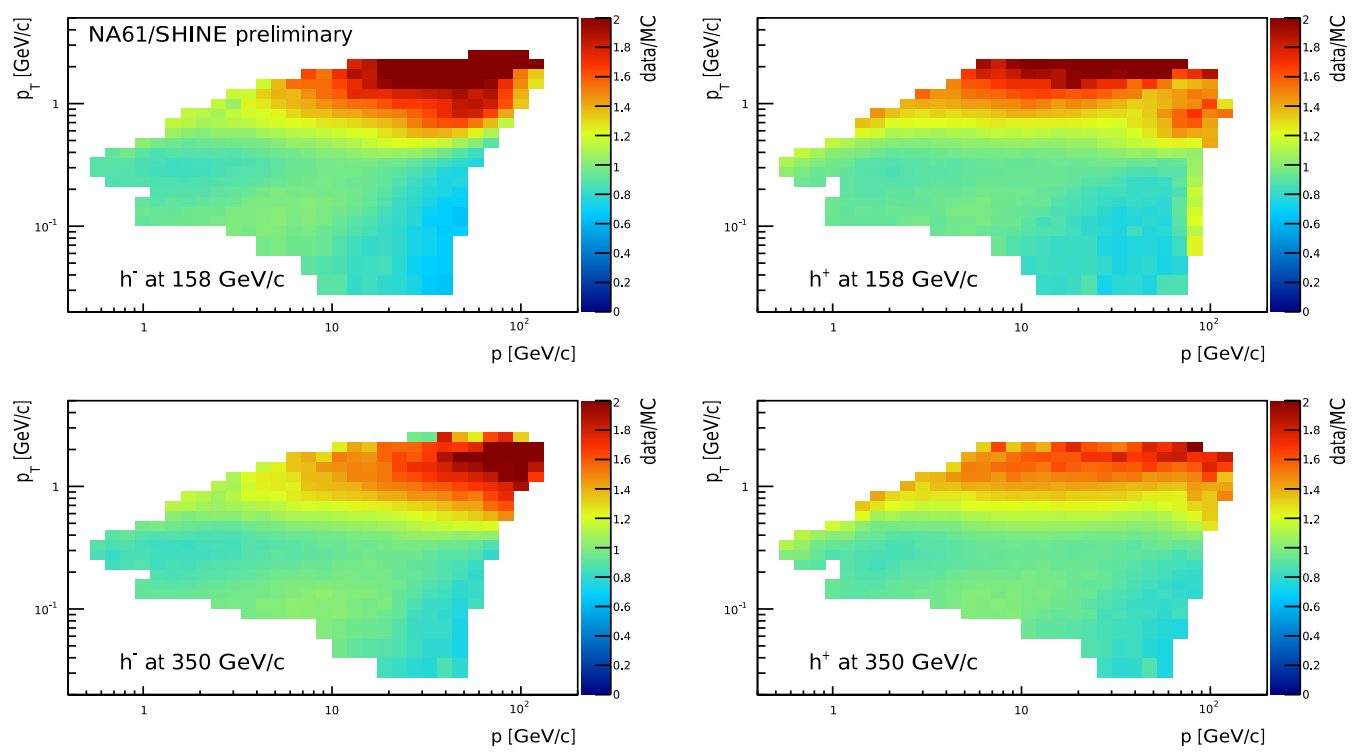

Figure 8. NA61/SHINE preliminary results: comparison of measured charged hadron production yields in $\pi^{-}+\mathrm{C}$ interactions to predictions from EPOS 1.99. Colors denote the ratio of data over MC.
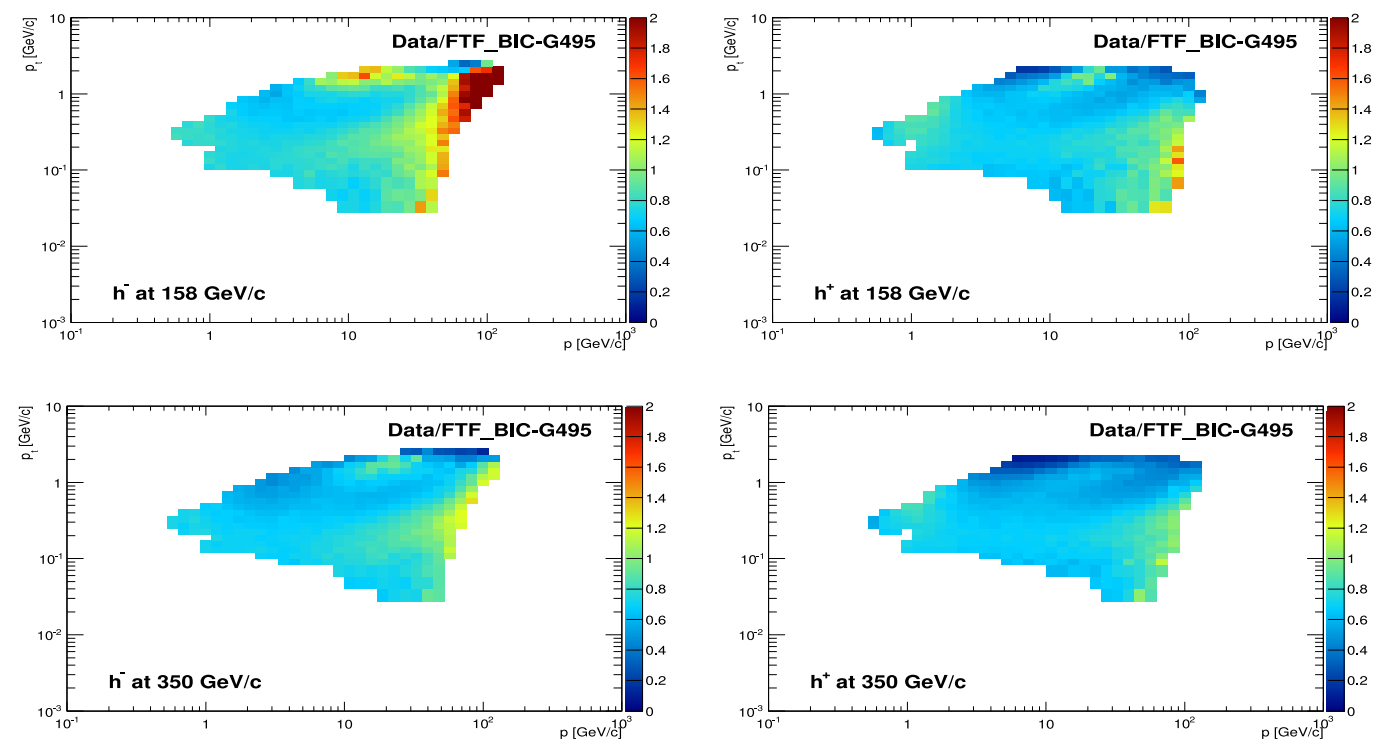

Figure 9. NA61/SHINE preliminary results: comparison of measured charged hadron production yields in $\pi^{-}+\mathrm{C}$ interactions to predictions from the FTF_BIC physics list in GEANT4.9.5. Colors denote the ratio of data over MC.
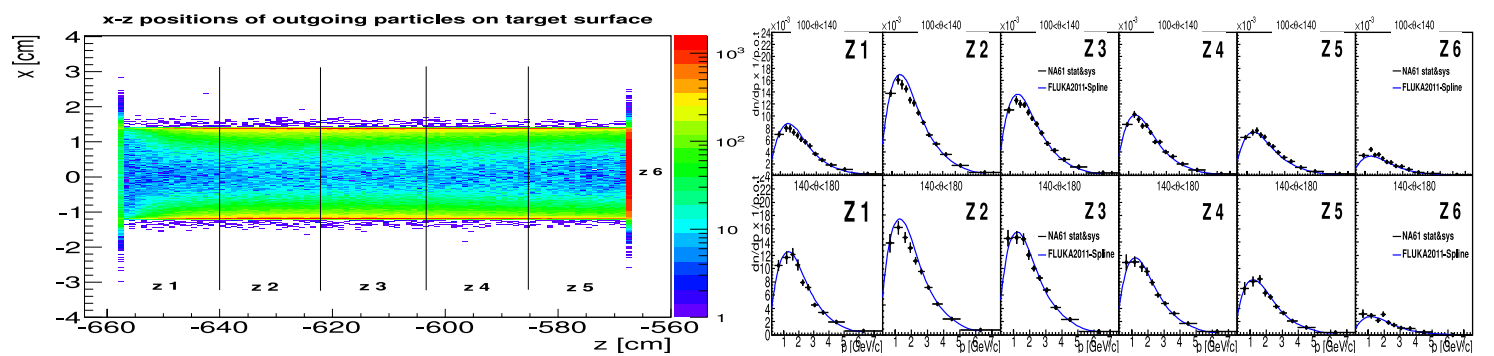

Figure 10. NA61/SHINE preliminary results (data 2009). Left: the $x-z$ distribution of outgoing tracks from the long target surface: 6 longitudinal intervals $\left(z_{1}\right.$ to $\left.z_{6}\right)$ used for the analysis are indicated on the plot. Right: fully corrected yields of $\pi^{+}$for two bins of polar angle $([100,140] \mathrm{mrad}$ and $[140,180] \mathrm{mrad})$ measured in 6 longitudinal intervals. The vertical error bars on the data points show the total (stat. and syst.) uncertainty. FLUKA2011 predictions are also shown. 


\section{Conclusions}

Fixed-target hadroproduction experiments (HARP, MIPP, NA49 and NA61/SHINE) have been reviewed.

The HARP experiment has already made important contributions to the cross section measurements relevant for cosmic ray and neutrino experiments.

Measurements of charged pion, kaon, proton, $K_{S}^{0}$ and $\Lambda$ production in $\mathrm{p}+\mathrm{C}$ interactions at $31 \mathrm{GeV} / \mathrm{c}$ released recently by NA61/SHINE are of significant importance and were already employed for precise predictions of the J-PARC neutrino beam used for the first stage of the T2K experiment. Preliminary results from the large-statistics 2009 run are presented here. These measurements together with preliminary results on hadron production in $\pi^{-}+\mathrm{C}$ interactions at 158 and $350 \mathrm{GeV} / \mathrm{c}$ are important for correct interpretation of data from cosmic ray experiments.

Fixed-target hadroproduction experiments provide also a large amount of input for validation and tuning of hadron production models in Monte-Carlo generators.

Thus, hadron production experiments have already contributed to the recent advances in cosmic rays and neutrino physics. Clearly, hadroproduction studies are a must for future precision cosmic ray and neutrino experiments.

It is a pleasure to thank the organizers of the ISVHECRI'2014 for the invitation to participate in this interesting conference and for the possibility to present the results described here. The author is grateful to HARP and NA61/SHINE colleagues.

\section{References}

[1] J. Abraham et al. [Pierre Auger Collaboration], Nucl. Instrum. Meth. A 523 (2004) 50 http://www . auger .org/

[2] T. Antoni et al. [KASCADE Collaboration], Nucl. Instrum. Meth. A 513 (2003) 490 http://www-ik.fzk.de/KASCADE_home.html

[3] R. Abbasi et al. [IceCube Collaboration], Nucl. Instrum. Meth. A 700 (2013) 188 http://icecube.wisc.edu/

[4] I. C. Mariş et al., Nucl. Phys. Proc. Suppl. 196 (2009) 86

[5] M.G. Catanesi et al. [HARP Collaboration], Nucl. Instrum. Meth. A571 (2007) 527

[6] J. Strait et al., Phys. Rev. ST Accel. Beams 13 (2010) 111001; http://www.hep.ph.ic.ac.uk/iss/

[7] M. Sajjad Athar, M. Honda, T. Kajita, K. Kasahara and S. Midorikawa, Phys. Lett. B 718 (2013) 1375; M. Honda et al., Phys. Rev. D83 (2011) 123001; ibid D70 (2004) 043008; G. D. Barr, T. K. Gaisser, P. Lipari, S. Robbins and T. Stanev, Phys. Rev. D70 (2004) 023006; G. Battistoni, A. Ferrari, T. Montaruli and P. R. Sala, [arXiv:hep-ph/0305208]; Astropart. Phys. 19 (2003) 269 [Erratum-ibid. 19 (2003) 291]; G. Battistoni, A. Ferrari, P. Lipari, T. Montaruli, P. R. Sala and T. Rancati, Astropart. Phys. 12 (2000) 315

[8] M. Bonesini and A. Guglielmi, Phys. Rept. 433 (2006) 65; S. Kopp, Phys. Rept. 439 (2007) 101
[9] M. H. Ahn et al. [K2K Collaboration], Phys. Rev. Lett. 90 (2003) 041801

[10] M. H. Ahn et al. [K2K Collaboration], Phys. Rev. D74 (2006) 072003

[11] A. A. Aguilar-Arevalo et al. [MiniBooNE Collaboration], arXiv:0704.1500 [hep-ex]; E. Church et al. [BooNE Collaboration], FERMILAB-PROPOSAL0898 (1997)

[12] A. A. Aguilar-Arevalo et al. [SciBooNE Collaboration], FERMILAB-PROPOSAL-0954 (2006)

[13] M. Anfreville et al., Nucl. Instrum. Meth. A481 (2002) 339

[14] M. Baldo-Ceolin et al., Nucl. Instrum. Meth. A532 (2004) 548

[15] M.G. Catanesi et al. [HARP Collaboration], Nucl. Phys. B732 (2006) 1

[16] M.G. Catanesi et al. [HARP Collaboration], Nucl. Instrum. Meth. A572 (2007) 899

[17] M.G. Catanesi et al. [HARP Collaboration], Eur. Phys. J. C52 (2007) 29

[18] M.G. Catanesi et al. [HARP Collaboration], Astropart. Phys. 29 (2008) 257

[19] M.G. Catanesi et al. [HARP Collaboration], Astropart. Phys. 30 (2008) 124

[20] M. Apollonio et al. [HARP Collaboration], Nucl. Phys. A821 (2009) 118

[21] M.G. Catanesi et al. [HARP Collaboration], Eur. Phys. J. C51 (2007) 787

[22] M.G. Catanesi et al. [HARP Collaboration], Eur. Phys. J. C53 (2008) 177

[23] M.G. Catanesi et al. [HARP Collaboration], Eur. Phys. J. C54 (2008) 37

[24] M.G. Catanesi et al. [HARP Collaboration], Phys. Rev. C77 (2008) 055207

[25] M. Apollonio et al. [HARP Collaboration], Phys. Rev. C80 (2009) 065207

[26] M. Apollonio et al. [HARP Collaboration], Phys. Rev. C80 (2009) 035208

[27] M. Apollonio et al. [HARP Collaboration], Phys. Rev. C82 (2010) 045208

[28] A. A. Aguilar-Arevalo et al. [MiniBooNE Collaboration], Phys. Rev. D79 (2009) 072002

[29] A. A. Aguilar-Arevalo et al. [MiniBooNE Collaboration], Phys. Rev. Lett. 98 (2007) 231801

[30] J. R. Sanford and C. L. Wang, "Empirical formulas for particle production in $\mathrm{p}$-Be collisions between 10 and $35 \mathrm{BeV} / \mathrm{c} "$, BNL, AGS internal report (1967)

[31] A. Bagulya et al., JINST 4 (2009) P11014

[32] M.G. Catanesi et al., JINST 3 (2008) P04007

[33] M. Apollonio et al. [HARP Collaboration], Phys. Rev. C80 (2009) 065204

[34] S. Agostinelli et al. [GEANT4 Collaboration], Nucl. Instrum. Meth. A506 (2003) 250; J. Allison et al., IEEE Transactions on Nuclear Science 53 (2006) 270

[35] N.V. Mokhov, S.I. Striganov, "MARS overview", FERMILAB-CONF-07-008-AD, 2007

[36] A. Fasso et al., CERN-2005-10 (2005); G. Battistoni et al., AIP Conf. Proc. 896 (2007) 31 http://www.fluka.org/

[37] K. Gallmeister and U. Mosel, Nucl. Phys. A826 (2009) 151; O. Buss et al., Phys. Rept. 512 (2012) 1 http://gibuu.physik.uni-giessen.de/GiBUU/ 
[38] https://edms.cern.ch/file/1184197/2/fluka 2011_harp_updated.pdf

https ://edms . cern.ch/file/1218221/1/fluka 2011_harp_ta.pdf

[39] http://hepdata.cedar.ac.uk/

[40] J. M. Paley et al. [MIPP Collaboration], Phys. Rev. D 90 (2014) 032001

[41] N. Graf et al. [MIPP Collaboration], Nucl. Instrum. Meth. A 615 (2010) 27

[42] T. S. Nigmanov et al. [MIPP Collaboration], Phys. Rev. D 83 (2011) 012002

[43] S. Afanasev et al. [NA49 Collaboration], Nucl. Instrum. Meth. A430 (1999) 210

[44] C. Alt et al., Eur. Phys. J. C45 (2006) 343; T. Anticic et al., Eur. Phys. J. C65 (2010) 9; T. Anticic et al., Eur. Phys. J. C68 (2010) 1

[45] C. Alt et al., Eur. Phys. J. C49 (2007) 897; G. Barr et al., Eur. Phys. J. C49 (2007) 919; B. Baatar et al., Eur. Phys. J. C73 (2013) 2329; O. Chvala et al., Eur. Phys. J. C73 (2013) 2364

[46] http://spshadrons.web.cern.ch/spshadrons

[47] P. Adamson et al. [MINOS Collaboration], Phys. Rev. Lett. 107 (2011) 021801; ibid 107 (2011) 181802; Phys. Rev. D 84 (2011) 071103

[48] L. Fields et al. [MINERvA Collaboration], Phys. Rev. Lett. 111 (2013) 2, 022501; G.A. Fiorentini et al. [MINERvA Collaboration], Phys. Rev. Lett. 111 (2013) 2, 022502

[49] N. Antoniou et al., CERN-SPSC-2006-034, CERN-SPSC-2007-004; N. Abgrall et al. [NA61/SHINE Collaboration], CERN-SPSC-2007019, CERN-SPSC-2007-033, CERN-SPSC-2008018, CERN-OPEN-2008-012, CERN-SPSC-2008026, CERN-SPSC-2009-001, CERN-SPSC-2009031, CERN-SPSC-2010-025, CERN-SPSC-2011005, CERN-SPSC-2011-035, CERN-SPSC-2012029, CERN-SPSC-2013-028

http://na61.web.cern.ch/na61/

[50] K. Abe et al. [T2K Collaboration], Nucl. Instrum. Meth. A659 (2011) 106; Phys. Rev. Lett., 107 (2011) 041801; Phys. Rev. D85 (2012) 031103; Phys. Rev. Lett., 112 (2014) 061802; Phys. Rev. Lett., 112 (2014) 181801 http://www.t2k.org/

[51] K. Abe et al. [T2K Collaboration], Phys. Rev. D87 (2013) 012001

[52] N. Abgrall et al. [NA61/SHINE Collaboration], Eur. Phys. J. C74 (2014) 2794

[53] N. Abgrall et al. [NA61/SHINE Collaboration], CERN-SPSC-2014-031 / SPSC-SR-145

[54] N. Abgrall et al. [NA61/SHINE Collaboration], JINST 9 (2014) P06005
[55] N. Abgrall et al. [NA61/SHINE Collaboration], Phys. Rev. C84 (2011) 034604

[56] N. Abgrall et al. [NA61/SHINE Collaboration], Phys. Rev. C85 (2012) 035210

[57] C. Alt et al. [NA49 Collaboration], Eur. Phys. J. C45 (2006) 343

[58] C. Strabel, Ph.D. Thesis, ETH Zurich, 2011; ETH19538

[59] T. Palczewski, Ph.D. Thesis, NCNR, Warsaw, 2012

[60] M. Posiadala, Ph.D. Thesis, Warsaw University, 2012

[61] S. Murphy, Ph.D. Thesis, Geneva University, 2012; CERN-THESIS-2012-093

[62] N. Abgrall et al. [NA61/SHINE Collaboration], "Measurements of $\pi^{ \pm}, K^{ \pm}, K_{S}^{0}, \Lambda$ and proton production in proton-carbon interactions at $31 \mathrm{GeV} / c$ with the NA61/SHINE spectrometer at the CERN SPS", paper in preparation

[63] L. Zambelli, Ph.D. Thesis, LPNHE, Paris, 2013

[64] N. Abgrall et al. [NA61/SHINE Collaboration], Phys. Rev. C 89 (2014) 025205

[65] K. Werner, Nucl. Phys. A525 (1991) 501c; Phys. Rep. 232 (1993) 87

[66] T. Pierog, this proceedings;

K. Werner, F.-M. Liu, T. Pierog, Phys. Rev. C 74 (2006) 044902

[67] S.M. Bass et al., Prog. Part. Nucl. Phys. 41 (1998) 225; M. Bleicher et al., J. Phys. G: Nucl. Part. Phys. 25 (1999) 1859

[68] V. Uzhinsky, arXiv:1308.0736 [hep-ph]; arXiv:1107.0374 [hep-ph]

[69] V. Uzhinsky, arXiv:1109.6768 [hep-ph]; arXiv:1404.2026 [hep-ph]

[70] https://edms.cern.ch/file/1186772/1/flu ka_vs_na61.pdf https://edms.cern.ch/file/ 1219646/1/gibuu. pdf https://edms . cern.ch/file/1250157/1/VMC_ internal_note_laura.pdf

[71] M. Haug, Messung des Wirkungsquerschnitts von Pion-Kohlenstoff-Wechselwirkungen mit Hilfe des NA61 Detektors, Diploma Thesis, Karlsruher Institut für Technologie, 2012

[72] M. Ruprect, Measurement of the Spectrum of Charged Hadrons in $\pi^{-}+C$ Interactions with the NA61 Experiment, Diploma Thesis, Karlsruher Institut für Technologie, 2012

[73] N. Abgrall, Ph.D. Thesis, Geneva University, 2011; CERN-THESIS-2011-165

[74] N. Abgrall et al. [NA61/SHINE Collaboration], Nucl. Instrum. Meth. A701 (2013) 99

[75] A. Hasler, Ph.D. Thesis, Geneva University, 2014 in preparation 